\title{
Capítulo 1 \\ Tendencias actuales en los estudios sobre tecnologías digitales y educación. Hacia una perspectiva culturalmente informada
}

n Lecciones de los maestros (2011), George Steiner afirma que el
arte de enseñar es, en esencia, el proceso de construir una comunidad sobre la base de la comunicación. Bajo esta premisa, las preguntas fundamentales que habría que hacer en términos educativos serían: ¿qué define el sistema comunicativo actual? y, en consecuencia, ¿qué tipo de comunidades de aprendizaje es posible construir? Dichas preguntas laten con fuerza en el debate sobre el presente y el futuro de la educación, que se desarrolla en el contexto de un peso creciente de la tecnología en la construcción y transmisión de conocimientos, percepciones y sensibilidades.

Habida cuenta de la centralidad de la tecnología en el discurso moderno sobre el cambio social y el progreso, era de esperarse que las discusiones sobre la mediación de las tecnologías digitales en los procesos de enseñanza y aprendizaje dieran lugar a debates y polémicas. En paralelo al proceso de domesticación social de Internet y sus parientes, ha tenido lugar un choque entre posturas que remeda las polémicas entre apocalípticos e integrados (Eco, 2004). Mientras 
unos consideran que las tecnologías digitales son herramientas de control social (Siegel, 2008), que igualan el acceso a la información con el conocimiento (Wolton, 2011) y que obstruyen nuestra capacidad para leer y pensar en profundidad (Carr, 2008), otros las reivindican como culminación material del ideal liberal-ilustrado de un aprendizaje más autónomo, complejo y colaborativo (Anderson, 2007; Johnson, 2011; Negroponte, 1995; Prensky, 2001).

En la versión actual del debate, ya está asumido que la llegada de las tecnologías digitales ha generado cambios sociales y educativos inevitables. Lo que se intenta evaluar ahora es, más bien, la naturaleza de esos cambios y las estrategias más adecuadas para hacerles frente. De ahí que, como se intentará mostrar a continuación, de un tiempo para acá se venga insistiendo en la pertinencia de un enfoque más sensible a los procesos de construcción social de sentido, que permita, entre otras cosas, una lectura crítica de las polémicas históricas que se han producido en torno a la tecnología.

El objetivo de este capítulo es, entonces, mostrar que en el campo de la investigación y la teorización sobre educación y tecnologías digitales, se está produciendo un giro comunicativo y cultural. En otras palabras, se trata de mostrar que la presencia de las tecnologías digitales en ámbitos escolares obliga a pensar la educación en los términos que plantea Steiner, es decir, poniendo en el centro a los actores y sus procesos de construcción de sentido. Para ello aludirá, en primer lugar, a algunos informes que señalan los desafíos de orden conceptual y actitudinal que quedan por resolver en contextos donde las tecnologías móviles han permitido reducir significativamente la brecha digital de primera generación. Luego se traerán a colación algunas ideas, recomendaciones y propuestas educativas provenientes de teóricos e investigadores de la cultura digital. Esto, para intentar reconstruir la trama de sugerencias y recomendaciones que muchas instituciones educativas toman como referencia en el diseño de políticas de enseñanza y aprendizaje mejor acopladas a 
un sistema comunicacional basado en Internet. Finalmente, se revisará el papel asignado a los jóvenes en algunas investigaciones sobre usos tecnológicos y prácticas mediáticas.

\section{Brecha cognitiva, tecnologías móviles y actitudes negativas}

Cada vez son más numerosos los estudios llevados a cabo por instituciones internacionales sobre el futuro de la educación en la era digital. Como se ha argumentado en trabajos anteriores (López, 2015), pareciera que dichos estudios adoptan como marco común el discurso de la sociedad-red (Castells, 1997, 1998a, 1998b, 2008; Lee \& Wellman, 2012), según el cual Internet ha sido decisivo para la organización social global en una lógica de redes. Prueba de ello son los informes que se mencionan a continuación, en los que, a partir de tal premisa sociológica, se analizan los retos y oportunidades de una educación digitalmente mediada.

El informe Hacia las sociedades del conocimiento (Unesco, 2005) constituye una primera referencia obligada. El texto no solo alude a un cambio de época, sino, incluso, de una segunda revolución industrial propiciada por las tecnologías digitales. De acuerdo con los autores de este documento, dicha revolución implica, básicamente, un aumento significativo de las capacidades de autonomía y libertad por parte de grupos e individuos involucrados en procesos educativos e investigativos. "El potencial ofrecido por la utilización razonable y resuelta de las nuevas tecnologías -reza este escrito- abre auténticas perspectivas al desarrollo humano y sostenible, así como a la edificación de sociedades más democráticas" (Unesco, 2005, p. 5). No obstante, el informe no solo alude a situaciones prometedoras, sino que también expone algunos escenarios inquietantes. 
Allí también se indica que es importante distinguir dos tipos de obstáculos en la conformación de redes inclusivas para la producción de nuevo conocimiento: por un lado, los que están relacionados con el acceso a las tecnologías digitales; por otro, los asociados a factores educativos, culturales y lingüísticos que siguen haciendo de Internet una herramienta de difícil comprensión y manejo. Este es, precisamente, uno de sus aportes más significativos: señalar la existencia de una brecha cognitiva, no material, que impide a muchas personas aprovechar el potencial transformador de Internet. De hecho, a juicio de la Unesco, la brecha cognitiva evidencia que la geografía del desarrollo se reproduce en el ciberespacio. De ahí que sea en el escenario de la educación y la competencia digital, y no tanto en el del desarrollo tecnológico, donde según el documento se adviertan las mejores posibilidades de reducir las desigualdades digitales.

En Directrices para las políticas de aprendizaje móvil (Unesco, 2013)', la Unesco integra las tecnologías móviles a la reflexión sobre la educación en las sociedades del conocimiento. En el documento se vuelve a insistir en la gravedad de la brecha cognitiva y se destaca en la urgencia de "nuevas conceptualizaciones de los modelos tradicionales de uso y aplicación de las tecnologías" (Unesco,

3 Los autores argumentan que dispositivos móviles como tabletas y teléfonos inteligentes, por ser económicos, portables y de alta oferta y demanda, son cada vez más utilizados por docentes y estudiantes en procesos de enseñanza-aprendizaje. Así, la popularidad de estos dispositivos los convierte en una variable de análisis ineludible tanto para el estudio de la relación educación-tecnologías digitales en la segunda década del siglo XxI, como para la formulación de políticas de aprendizaje digitalmente mediado. El informe también afirma que, pese a la diferencia significativa en términos de acceso y uso de telefonía móvil entre países desarrollados y países en desarrollo (4 de cada 5 personas en países desarrollados vs. 2 de cada 5 personas en países en desarrollo), "se calcula que, para 2017, aproximadamente la mitad de la población de los países en desarrollo tendrá al menos una suscripción activa por persona a un teléfono móvil” (Unesco, 2013, p. 7). Con lo cual, frente al problema de la brecha digital, surgirían nuevas dimensiones de análisis y oportunidades para reducirla. 
2013, p. 8). Además de ponderar las ventajas estratégicas del uso de dispositivos como tabletas y celulares ${ }^{4}$ para la reducción de la brecha de acceso a tecnologías digitales, se presentan algunas recomendaciones con talante decididamente formativo, tales como: invertir no solo en la adquisición de nuevas tecnologías sino también en patrocinar iniciativas educativas orientadas a un uso creativo de las mismas; integrar las tecnologías móviles a los programas de capacitación profesional de los docentes; fortalecer la comunicación entre educadores y educandos, y eliminar la prohibición generalizada de dispositivos móviles en las aulas.

Sin embargo, más allá de recomendaciones que pueden llegar a ser de sentido común en el ámbito educativo, llama la atención que se insista en la raíz cultural de la brecha cognitiva. En el informe se insiste en la necesidad de promover campañas para transformar los significados y las actitudes sociales negativas con respecto a los dispositivos móviles. A juicio de los autores, hace falta que instituciones, maestros y estudiantes estén en capacidad de reconocer en la nueva generación de dispositivos tecnológicos instrumentos para la educación y no sólo para el entretenimiento. De hecho, en el documento se hace referencia en los siguientes

4 El informe aporta las siguientes razones para preferir las tecnologías móviles como alternativa a los ordenadores:

- Las tecnologías móviles son, a diferencia de los ordenadores, portables y baratas. Por tanto, los proyectos de aprendizaje a través de móvil podrían sustentarse sobre el supuesto del acceso ininterrumpido y no regulado del estudiante a la tecnología.

- Actualmente, las tecnologías móviles y sus redes de telecomunicaciones penetran regiones de dificil acceso para instituciones educativas y bibliotecas. Ello, junto al precio cada vez menor de los teléfonos inteligentes, garantizaría un grupo de usuarios más diverso y familiarizado con el uso de estas tecnologías.

- La tecnología móvil y la computación por aplicaciones brinda un mayor grado de personalización y adaptación a las necesidades individuales.

- Finalmente, móviles y tabletas posibilitarían una suerte de enseñanzaaprendizaje que se expande más allá del aula. 
términos a las barreras no materiales que impiden aprovechar el potencial transformador de estos dispositivos:

Las actitudes sociales negativas en relación con las posibilidades pedagógicas de la tecnología móvil constituyen la barrera más inmediata para la aceptación generalizada del aprendizaje móvil. En términos generales, se suele pensar en los dispositivos móviles (y los teléfonos móviles, en particular) como medios de entretenimiento, no de educación, por lo que este tipo de tecnología a menudo se descarta en las aulas por considerar que supone una distracción o un trastorno. Históricamente, el tamaño pequeño de las pantallas y los peculiares métodos de introducir información en los dispositivos móviles se han considerado también desventajas para su empleo en la enseñanza. (...) los encargados de formular políticas también pueden tomar medidas para educar a la población sobre las ventajas del aprendizaje móvil. (Unesco, 2013, p. 39)

Por su parte, en los reportes Horizon se presenta un panorama similar, donde los retos por enfrentar son de índole predominantemente cultural. Desde las versiones de 2012 (Johnson, Adams, \& Cummins, 2012), se alude a la emergencia de las tecnologías móviles y el aprendizaje informal como factores ineludibles en el debate sobre el presente y el futuro de la educación. A modo de ejemplo, en el reporte de 2013 (Johnson et al., 2012) se afirma que el aprendizaje mediado por dispositivos digitales ha pasado de ser un acontecimiento periférico a una tendencia dominante, por lo que colegios y universidades deben estar en la capacidad de anticipar y adaptarse a los cambios que esto conlleva. Para ello

5 El Horizon Report es una publicación anual patrocinada por el New Media Consortium. En estos documentos se intenta delinear el paisaje de las nuevas tecnologías para la enseñanza, el aprendizaje y la investigación, de modo que sus resultados sean útiles para educadores y tomadores de decisión. Los resultados de los informes se obtienen a partir de la metodología Delphi en la que participan grupos de expertos sobre tecnologías y tendencias educativas. Para más información, véase http://www.nmc.org/horizon-project 
es imprescindible tomar como primera referencia las actitudes de profesores, estudiantes y directivos, así como las políticas educativas al interior de cada institución. Esto porque tales redes invisibles de normas y significados constituyen, según los autores, el fondo sobre el que deben impulsarse estrategias innovadoras de alfabetización mediática y adopción de nuevas tecnologías.

Sin perder de vista la muy cuestionable concepción mercantil de la educación superior que late en cada uno de los reportes Horizon, habría que mencionar también la relación que en ellos se establece entre cambios de paradigma educativo y estrategias de innovación cultural. Dicha relación se advierte de forma especialmente clara en sus versiones más recientes, donde se afirma que la educación superior debe adaptarse a formas de aprendizaje híbrido y colaborativo, potenciados por la adopción masiva de tecnologías móviles y el crecimiento exponencial de contenidos abiertos. En ese contexto, se hace referencia a una "cultura del cambio y la innovación” (Johnson, Adams Becker, Estrada, \& Freeman, 2014, 2015; Johnson et al., 2013) de extracción empresarial, que debiera exportarse a la educación superior, a fin de acelerar la adopción de nuevas tecnologías. De ese modo, se concluye, las universidades podrían ser instituciones más eficientes y mejor articuladas con el sistema productivo de las sociedades actuales.

A modo de síntesis, podría decirse que tanto los informes de la Unesco como las distintas versiones del Horizon Report coinciden en señalar un cambio en curso de paradigma educativo. Dicho cambio estaría propiciado por las tecnologías digitales, pero necesitaría, de un cambio conceptual y actitudinal para acabar de hacerse efectivo. Por un lado, las tecnologías móviles son descritas como una solución efectiva a la brecha digital de primera generación, pues su precio, prestaciones y popularidad permitirían dar un salto hacia experiencias de aprendizaje personalizado y expandido mucho más allá del aula. Por otro, se insiste en que para poder aprovechar su potencial se necesita un cambio de mentalidad, en 
virtud del cual estos dispositivos dejen de estar asociados únicamente a la distracción y al entretenimiento.

Vale destacar, entonces, que al tiempo que teléfonos inteligentes, ultrabooks y tabletas figuran como solución a la brecha digital de primera generación (acceso), van adquiriendo protagonismo todos aquellos obstáculos no materiales (actitudes, políticas institucionales, creencias, idioma, etc.) que impiden aprovechar de mejor manera las tecnologías digitales. Naturalmente, los informes aquí reseñados no son los únicos que insisten en este tipo de desafíos culturales; también lo hacen, a su manera, los autores y trabajos que se presentan a continuación.

\section{La investigación en educación y medios digitales: Entre las mediaciones críticas y la cultura web 2.0}

En esta sección, se hará alusión a los trabajos de algunos investigadores de la cultura digital especialmente influyentes en el campo de las políticas educativas. Tomándolos como referencia, se intentará evidenciar un cambio de perspectiva a la hora de estudiar el papel de las tecnologías digitales en la educación. De la pregunta por el acceso a las tecnologías digitales se ha pasado a la preocupación por la seguridad informática y, en una fase más reciente, al problema de los entramados culturales que informan las prácticas mediáticas juveniles.

Autores como Sonia Livingstone (2004) y David Buckingham (2005) insistieron en su momento en que las instituciones educativas debían ocuparse, ante todo, de diseñar e implementar modelos de alfabetización y acompañamiento parental dirigidos a garantizar unas prácticas mediáticas más seguras e incluyentes. Según esto, no había que conformarse únicamente con llevar tecnologías digitales a las aulas. Una vez establecida la conexión inicial, las instituciones educativas debían establecer protocolos de seguridad 
orientados a minimizar el riesgo informático. Conseguido este objetivo, lo siguiente sería identificar y afrontar las desigualdades sociodemográficas que afectaban los niveles de participación y comunicación de sus estudiantes.

Desde esa perspectiva, la principal responsabilidad de padres y maestros consistía en proveer definiciones, horizontes de comprensión y nociones de riesgo a los más jóvenes, de tal forma que estos últimos se formasen un criterio sobre los peligros que debían enfrentar al navegar por Internet. Dando por sentado que jóvenes y niños contaban con un nivel aceptable en el uso de dispositivos digitales, había que generar en ellos una conciencia aguda de los riesgos a los que podían estar expuestos.

Más adelante, Cobo (2010) aportará una crítica fundamental para dar un giro a la discusión. A su juicio, pareciera que muchas escuelas, intelectuales e investigadores padecen una suerte de fetichismo tecnológico, pues se asume erróneamente que la inversión en infraestructura equivale a hacer frente al reto de la revolución digital. De ahí que el mismo autor se pregunte si no se habrá sobreestimado a las TIC frente a desafíos más complejos, como el desarrollo de competencias que permitan gestionar la información y producir conocimientos socialmente relevantes.

Según Cobo, fruto del excesivo valor que por años se ha asignado al objeto tecnológico, directivos, maestros y hacedores de política educativa han terminado inmersos en el falso dilema de la conexión vs. la desconexión de la escuela. En otras palabras, la conectividad ha sido asumida como un valor esencialmente positivo mientras que, por el contrario, la desconexión ha terminado vinculada al analfabetismo y la ignorancia. Esto explicaría, entonces, por qué muchas escuelas han entrado en una espiral de consumo tecnológico que ha terminado por separarlas de su misión fundamental: generar las mediaciones críticas necesarias para hacer de las tecnologías digitales una herramienta de cambio social y educativo. 
Entre tanto, postulan Cobo y Moravec (2011), el mundo ha cambiado y el nuevo sistema comunicativo obliga, más bien, a mirar más allá del objeto tecnológico. En este sentido, se debe enfatizar en la capacidad de generar, conectar y diseminar el conocimiento creado. En este nuevo orden de problemas, adquieren especial relevancia aquellos lugares otrora invisibles para la educación tradicional, pero que se han convertido en ámbitos de aprendizaje tremendamente significativos en el mundo juvenil. Según los autores, en la actualidad los jóvenes aprenden con herramientas y en contextos que desde el punto de vista adulto no tienen valor pedagógico. De hecho, argumentan los autores, por estar concentrada en mejorar sus indicadores de adquisición y uso tecnológico, la escuela no ha sabido ver que sus estudiantes habitan ya en el ciberespacio, que aprenden con los videojuegos y que socializan y se forjan una identidad en las redes sociales.

El trabajo de Davidson y Goldberg (2009) coincide con estos señalamientos en procura de un abordaje menos dependiente del dato tecnológico y mucho más atento a la comunicación y el juicio reflexivo. Según ellos, las instituciones educativas del siglo veintiuno tienen la misión histórica de promover en sus estudiantes una visión crítica no solo frente a sus propias prácticas mediáticas, sino también ante los valores que ordenan el desarrollo tecnológico. Afirman, además, que sobre la escuela recae la responsabilidad de promover formas de comunicación y sociabilidad más horizontales y descentralizadas. Esto con el fin de potenciar un espíritu de cooperación, creatividad e innovación que, al estar inspirado en Internet, cada día resulta más importante.

Vale mencionar que las posturas de Davidson y Goldberg también destacan por su tono ciberutópico. Mirando el panorama educativo actual a la luz de la filosofía de la web 2.0 (O’ Reilly, 2005), los autores argumentan que se vienen cambios radicales e inevitables. Según ellos, la actitud proactiva que se requiere para tener un lugar en el sistema comunicativo actual no podrá sino 
entrar en conflicto con cualquier modelo de escuela autoritario y estandarizado, como el tradicional, en el que el conocimiento se impone de arriba hacia abajo. Como resultado de ese choque de culturas, del crecimiento exponencial de la información disponible en la red y de la ubicuidad del acceso a las fuentes de información, la autoridad del maestro entrará en crisis y, en cambio, resultarán fortalecidos todos aquellos modelos de aprendizaje basados en la meritocracia, como los de las comunidades de internautas. De este modo, concluyen los autores, las experiencias de aprendizaje no formal, propias del ciberespacio, permearán definitivamente los muros de la escuela, al tiempo que permitirán a los estudiantes erigirse como miembros activos de una cultura de código abierto y protagonistas de un aprendizaje constante y ubicuo.

Por su parte, los trabajos de Jenkins (2008, 2009a, 2009b; Jenkins, Ford, \& Green, 2013) insisten en que el reto principal que debe enfrentar la educación va mucho más allá de lograr un uso competente en tecnologías digitales. Este consiste, más bien, en acompañar el tránsito en curso de una cultura de espectadores a una cultura de la participación. Con base en sus estudios sobre comunidades juveniles online, Jenkins afirma que una buena educación mediática debería preparar para moverse con fluidez en una cultura de código abierto, en la que la producción social del significado es más que la suma de interpretaciones individuales. De ahí la importancia de un diálogo entre toda la comunidad educativa sobre las habilidades y competencias sociales que se deben promover ${ }^{6}$.

6 En su estudio sobre los retos actuales de la educación mediática (Jenkins, 2009b), el autor propone trabajar en el desarrollo de las siguientes habilidades: experimentar con el entorno como forma de resolver problemas (Play); adoptar identidades alternativas con el fin de improvisar y descubrir; interpretar y construir modelos dinámicos de los procesos del mundo real; analizar el ambiente y cambiar el foco de atención a detalles novedosos; mezclar y combinar contenidos mediáticos de manera significativa; interactuar significativamente con herramientas que expanden las capacidades mentales; 
Para Jenkins, el proceso de imaginar la educación que necesitan las sociedades contemporáneas no implica necesariamente una ruptura con la tradición, sino más bien una superación del egoísmo, la indiferencia y el aislamiento. Según él, la mejor versión de la cultura digital es aquella en la que las personas desarrollan su potencial cooperativo en plena conciencia de los flujos culturales que los circundan. La alfabetización mediática, por poner un ejemplo, no se presenta como una superación de la alfabetización textual, sino como un intento de ampliar horizonte de lo formativo, sin olvidar en ningún momento que leer y escribir siguen siendo recursos básicos para que el estudiante cultive su conciencia histórica y se forme una imagen coherente del mundo. En ese orden de ideas, Jenkins insiste en la importancia de evaluar los modos en que las sociedades, y en especial las comunidades educativas, se han venido relacionando con las tecnologías.

Por último, el autor afirma que, pese a la horizontalidad que supuestamente define las cibercomunidades, las figuras de liderazgo y autoridad (parental y docente) siguen siendo fundamentales para jóvenes y niños. A su juicio, una cultura de código abierto mal entendida puede degenerar en un liberalismo educativo tan ingenuo como peligroso (Jenkins, 2009b), en el que se asuma, sin evidencia alguna, que los jóvenes: (1) participan activamente en los procesos formativos, (2) reflexionan constantemente sobre los usos que le dan a las tecnologías y (3) están, por ende, en perfecta capacidad de generar un código ético para socializar y aprender en un entorno social tan complejo y diverso como el ciberespacio.

Una mirada panorámica a las ideas reseñadas en esta sección permite entrever un intento consciente por superar visiones

construir conocimiento de forma colaborativa; evaluar la confiabilidad y credibilidad de diferentes fuentes informativa; seguir el flujo de historias e información a través de múltiples modalidades e interactuar con distintas comunidades de forma respetuosa y crítica. 
excesivamente centradas en la adquisición de dispositivos y privilegiar, más bien, la formación de un juicio crítico y el desarrollo de competencias para comunicar y generar conocimiento en el marco de una cultura participativa. Se advierte, no obstante, falta de consenso frente al rol de padres y maestros. Pareciera que entre más se reivindica la idea de las tecnologías digitales como herramientas que dotan de autonomía a los jóvenes, más se insiste, por otra parte, en la relevancia de figuras de autoridad que les ayuden a orientar sus prácticas mediáticas en función de objetivos educativos e investigativos.

En cualquier caso, los planteamientos recogidos aquí dejan entrever que el rol asignado a los jóvenes en la reconfiguración del panorama educativo y cultural es decididamente protagónico. Dado que su capacidad para domesticar tecnologías digitales se da por sentada, pareciera que el joven es una figura de singular relevancia estratégica y simbólica. Esto justifica dedicar la siguiente sección a revisar qué papel se ha venido asignando a los jóvenes en el discurso sobre la revolución digital.

\section{Los jóvenes como agentes mediáticos y protagonistas del cambio educativo}

Ya sea por la creciente influencia del individualismo en red (Castells, 2008; Lee \& Wellman, 2012), por el fortalecimiento de las sociologías postindustriales más centradas en los sujetos culturales (Touraine, 2004, 2007), por una estrategia comercial interesada en asociar la juventud a lo nuevo de las nuevas tecnologías (Molinuevo, 2008) o por el juvenismo contemporáneo que según Maffesoli (2014) define el mundo actual, lo cierto es que cada vez más se asume que los jóvenes son participantes activos en el ecosistema comunicativo y no solo simples consumidores de medios. Siguiendo a del Río (2006), se podría afirmar que la pregunta no es ya ¿qué hacen las 
tecnologías digitales con las nuevas generaciones?, sino, más bien, ¿qué hacen las nuevas generaciones con las tecnologías digitales?

El giro más reciente de los dispositivos a los usuarios ha permitido, además, que se fortalezcan tentativas de análisis interesadas no solo en registrar, describir y caracterizar ciertas prácticas mediáticas juveniles, sino también en interpretarlas como parte de un circuito cultural. Además de intentos por determinar si existen cambios en los hábitos de consumo mediático (López-Vidales, González Aldea, \& De la Viña, 2011), de mediciones comparadas sobre usos de redes sociales (Bravo Ramírez, \& de Pablos Pons, 2013; Martínez, Fonseca, \& Esparcia, 2013) y de análisis de protocolos de comunicación en contextos de riesgo informático (Cáceres-Zapatero, Brändle Señán, \& Ruíz San-Román, 2013), actualmente se vienen desarrollando cada vez más estudios sobre las representaciones, normas y vínculos intangibles que informan las prácticas mediáticas juveniles en contextos lúdicos, políticos y académicos. Muestra de ello es la investigación de Skoric y Kwan (2011) sobre los procesos de socialización juvenil a través de redes sociales y juegos online multiplayer, el texto de Conroy, Feezell y Guerrero (2012) sobre la relación entre el establecimiento de grupos en Facebook y la calidad de la discusión política o la investigación de Kahne (Kahne, Lee, \& Feezell, 2011; Kahne, Middaugh, Lee, \& Feezell, 2011) sobre Internet y los compromisos cívicos de los jóvenes estadounidenses. Por consiguiente, a fin de entender cómo es que se ha producido este cambio de perspectiva, conviene revisar con más detalle algunos trabajos que han marcado un rumbo hacia descripciones más profundas del universo juvenil actual.

El trabajo de Carrie James (2009) constituye una primera referencia clave. En principio, la autora aporta una interesante clasificación de las actividades que los jóvenes llevan a cabo en la red. Según ella, se debe distinguir entre actividades que están asociadas a la autoexpresión y la experimentación con la propia identidad -tales como el diseño de avatares, la creación y circulación de 
contenidos y el mantenimiento de perfiles en redes sociales-, las que están orientadas a la consecución de fines asociativos - como las charlas online entre amigos o desconocidos-, las prácticas de consumo cultural y de entretenimiento - como descargar música, ver vídeos o jugar en línea- y, por último, aquellas relacionadas con la educación, la construcción de conocimiento y el compromiso cívico. Tal clasificación permite a James argumentar, primero, que los jóvenes no tienden a adoptar una actitud de consumo pasivo frente a los medios digitales y, segundo, que muchas de sus actividades en la red estarían orientadas por una serie de lenguajes, narrativas, códigos morales, rituales y sentimientos. Entonces, a fin de profundizar en la comprensión de las prácticas mediáticas juveniles, James plantea la importancia de identificar y reconstruir aquellos "espacios de afinidad" (Gee, 2005), que sirven como escenario para las actuaciones juveniles en la red.

Los trabajos de Ito y colaboradores (Ito, 1997; Ito, 2008; Ito, Daisuke, \& Misa, 2005; Ito et al., 2008; Ito et al., 2013) apuntan en una dirección similar. Llevados a cabo desde una premisa etnográfica, han contribuido decisivamente al fortalecimiento de una tendencia de análisis donde las prácticas mediáticas se explican a la luz de experiencias intersubjetivas, a partir del propio lenguaje juvenil y prestando mucha atención a la vida cotidiana de los participantes, en especial a sus tránsitos entre lo doméstico y lo escolar y entre el mundo online y offline.

En contravía de quienes asocian las tecnologías digitales con el advenimiento de una aldea global en la que el contacto cara a cara, los vínculos locales y la identidad colectiva son cada vez menos importantes, Ito et al. (2008) postulan que la presencia de los jóvenes en la red es, en realidad, un reflejo de su vida fuera de ella. A juicio de los autores, lo que los jóvenes hacen en la red está informado en gran medida por los gustos y las relaciones que establecen en la escuela y en el barrio. Si se analizaran desde una perspectiva etnográfica los usos que dan a sus celulares o el tipo de 
actividades que llevan a cabo en redes sociales, muy posiblemente se encontraría que dichas prácticas están orientadas a reforzar los lazos de amistad o a desarrollar sus intereses, pasatiempos y aficiones particulares. En este sentido, para los jóvenes Internet significaría mucho más que una mera herramienta informática; tendría mucho más que ver con una plataforma y una herramienta con la que superar el alejamiento físico, suplir necesidades afectivas, ampliar su red de contactos y ensanchar el horizonte de sus aspiraciones, intereses y pasatiempos particulares.

Por otra parte, sostienen Ito et al. (2008), si bien las actividades que los jóvenes llevan a cabo en el universo digital no parecen estar orientadas por una referencia parental o adulta directa, es frecuente que las brechas simbólicas entre el mundo adulto y el juvenil lleven a los jóvenes a desarrollar prácticas de resistencia ${ }^{7}$. Si desde la perspectiva juvenil pasar el rato con los amigos es una actividad necesaria y gratificante, puede que para padres y maestros esta sea vista como una pérdida de tiempo. En este orden de ideas, y en el caso de que los procesos de negociación intergeneracional llegasen a fallar, los jóvenes desarrollarían maneras de subvertir las barreras institucionales, sociales y técnicas impuestas a esta actividad haciendo uso, sobre todo, de herramientas y plataformas digitales.

No obstante, pese a que pudiera parecer que la brecha simbólica intergeneracional es infranqueable, Ito et al. (2008) recuerdan que existen casos exitosos (véanse Chávez \& Soep, 2005, 2010) de trabajo colaborativo entre jóvenes y adultos. En todos ellos, comentan, lo más importante ha sido estar en disposición de intercambiar experiencias, negociar marcos de significado comunes y

7 Esta línea de análisis recuerda los planteamientos de Mead (1977) sobre las culturas juveniles de talante prefigurativo, en las que "los pares reemplazan a los padres, instaurando una ruptura generacional sin parangón en la historia” (Martín-Barbero, 2002b). 
distribuir responsabilidades equitativamente. En la línea de estos autores se podría concluir que en la formulación de políticas de alfabetización mediática no se puede prescindir de un estudio juicioso de los contextos de participación que resultan significativos para los jóvenes. De ahí la importancia de una etnografía de los mundos juveniles.

Por último, la obra de Carles Feixa (2014) constituye una referencia mucho más atenta a la situación latinoamericana y colombiana. El autor propone un estudio interpretativo sobre la condición juvenil a partir de referencias culturales. Valiéndose de tres imágenes literarias (Tarzán, Peter Pan y Blade Runner) y de tres metáforas temporales (el reloj de arena, el analógico y el digital), Feixa realiza un paneo histórico sobre los marcadores identitarios socialmente construidos que han caracterizado las generaciones jóvenes para, finalmente, mapear los mundos sociales en los que habitan los jóvenes contemporáneos.

Feixa plantea dos tesis especialmente relevantes para la presente investigación: en primer lugar, que la juventud ha sido marcada históricamente por los adultos a través de símbolos poderosos y arbitrarios; en segundo lugar, que pese a lo anterior - es decir, pese a las clasificaciones simplistas diseñadas por los adultos- los jóvenes habitan actualmente en mundos plurales, en donde la hibridación cultural es la norma. En otras palabras, según él, tanto la identidad juvenil como las etiquetas que socialmente se han dispuesto para definir las generaciones más jóvenes resultan, al día de hoy, ambivalentes y conflictivas. Esto debido a que en el universo juvenil se yuxtaponen, e incluso se enfrentan, el espacio global y el espacio local, el tiempo real y el tiempo virtual, y el sedentarismo y el nomadismo. Valdría la pena preguntarse, entonces, si lo digital basta para definir la experiencia juvenil actual.

Los planteamientos de Feixa (2014), además de teóricamente relevantes, resultan metodológicamente sugestivos. En la línea de los autores antes citados, el autor invita a asumir una posición 
investigativa cimentada en la premisa de que las prácticas mediáticas y tecnológicas de los jóvenes/estudiantes son textos sociales que requieren ser interpretados. En este sentido, ahondar el fenómeno digital desde la perspectiva juvenil equivaldría a un ejercicio de reconstrucción hermenéutica a partir del contexto social y de la cultura juvenil, pues las tecnologías permiten una forma de estar en el mundo, pero también de imaginarlo.

En suma, los autores reseñados en este apartado permiten trazar una línea de análisis que va desde la taxonomía de usos tecnológicos y prácticas mediáticas hasta la reconstrucción hermenéutica y la interpretación. Se advierte aquí también una tentativa de giro cultural, donde los entramados de significado que dan sentido a las acciones de los sujetos se antojan cada vez más relevantes. De paso, Internet y sus parientes empiezan a figurar como algo más que meros instrumentos de consumo cultural o de interacción funcional. Los planteamientos más actuales indican, además, que las tecnologías digitales requieren ser estudiadas como símbolos y marcadores identitarios, es decir, desde una perspectiva iconográfica (Alexander, Bartmanski, \& Giesen, 2012).

Dado que el universo juvenil resulta esencial para la comprensión de la era digital y los retos que esta lanza a la educación, habría que preguntarse, ya en el plano investigativo, cuál es el rol que se debe asignar a las comunidades juveniles. Los estudios aquí reseñados han insistido en los peligros de reducir a los jóvenes a meras audiencias, usuarios o consumidores de tecnologías digitales. Su importancia estratégica, en el nivel del discurso sobre la revolución digital, señala la importancia de tomarlos como miembros de una o varias comunidades, como actores simbólicos y, además, como intérpretes culturales. Hoy más que antes es imprescindible escucharlos, explorar su mundo interior y discutir con ellos sobre los alcances simbólicos de sus acciones. Para ello, se requiere de una teoría de la acción que no se limite a la explicación racional de sus actuaciones en una lógica de costo-beneficio. Más bien, habría 
que aproximarse al estudio de las prácticas mediáticas juveniles por la vía de sus motivaciones no materiales, emocionales y morales.

También, queda claro que se debe seguir investigando acerca de las brechas intergeneracionales de sentido. Todo indica que este asunto está tremendamente conectado tanto con la brecha cognitiva a la que se aludía en la sección 1.1 como con el asunto de las mediaciones críticas que se abordó en la sección 1.2. De hecho, las supuestas diferencias entre la mirada juvenil y la mirada adulta constituyen otro indicador más de que el destino de las tecnologías digitales en la escuela dependerá de un conflicto cultural que se libra, como sugirió Castells (2008), en la mente de las personas.

A modo de cierre de este capítulo, cabe afirmar que los informes, investigaciones y reflexiones reseñados hasta aquí dejan dos lecciones sobre el tipo de investigación que es necesario emprender a fin de ensanchar el horizonte de comprensión sobre el fenómeno digital. La primera lección tiene que ver con la pertinencia de un enfoque teórico-metodológco que dé prioridad a aquellos asuntos comunicativos, simbólicos y disursivos, que permitan explicar mejor los procesos de apropiación social de las tecnologías digitales, sobre todo en la escuela. De ello dan cuenta buena parte de los documentos citados hasta aquí, pues la pregunta por las actitudes sociales, las lógicas del aprendizaje colaborativo, los rituales de socialización, los procesos de significación y los aspectos emocionales que enmarcan las prácticas mediáticas de los educandos adquieren relevancia. Podría decirse, entonces, que al tiempo que celulares y demás dispositivos portátiles han sido decisivos para resolver el problema del acceso, las dimensiones socioculturales del problema han ido emergiendo cada vez con más fuerza y claridad.

La segunda lección, relacionada también con la importancia de mirar más allá de los objetos materiales, consiste en poner a las personas en el centro del análisis, pero no solo en calidad de usuarios o consumidores. Efectivamente, hay un interés creciente por lo que hacen los jóvenes en Internet y por los usos que dan a 
celulares, redes sociales y demás. No obstante, vale recordar también que si desde la perspectiva más entusiasta se alaba la capacidad de los jóvenes para adaptarse al nuevo sistema comunicativo e impulsar una cultura de la participación, hay quienes no dejan de advertir sobre los peligros de idealizar la juventud. Por tanto, para evitar caer en exageraciones o simplificaciones, e incluso para someter a crítica el rol que desempeña la juventud en los discursos sobre la revolución digital, se hace necesario un abordaje teórico y metodológico donde el diálogo, los testimonios y las experiencias juveniles tengan mucho más protagonismo. Se trata, en cualquier caso, de escuchar atentamente a los personajes principales de un relato que parece moldear el tiempo presente. 